\title{
THE DELPHI METHOD AS APPLIED TO PUBLIC INVESTMENT DECISIONS: COMMENT
}

\author{
John R. Kaatz
}

\author{
Georgia Institute of Technology
}

The use of the Delphi Method in dealing with the problems of regional development planning and policy seems an appropriate application of this technique. As Helmer states, "Expert opinion must be called on whenever it becomes necessary to choose among several alternative courses of action in the absence of an accepted body of theoretical knowledge that would clearly single out one course as the preferred alternative. "l Certainly the dynamics of the problem attacked by Gunther and Vallery satisfy the conditions that Helmer specifies for the use of the technique. Moreover, the potential contribution of this approach in dealing with the economic development problem should not be overlooked. Looking at the record of success to date, in dealing with the economic development problem, some innovative effort appears warranted.

My reservations with respect to the paper given by Gunther and Vallery stem, therefore, not from the use of the Delphi technique per se, but rather from some of the details of its proposed application. Specifically, these are:

(1) The statement of the problem appears to lack sufficient precision.

(2) The "experts"selected (or the OEDP Committee) may not have adequate knowledge or predictive reliability.

(3) A non-symmetric, as opposed to a symmetric, panel of experts seems to be assumed, but this is given no explicit consideration.

(4) The structure of the questionnaire itself appears lacking with respect to the stated objective.

Under the first point, the proposed format suggests a group learning program in regional economics, and even if the present state of the science (or art) could be reached by the panel, it would probably take an inordinately long time. Perhaps a better approachwould be to first transform this overall objective into separate considerations of the alternative courses of action open to the communities involved, i.e., manufacturing development, nonmanufacturing development, etc., using separate, though not necessarily mutually exclusive, panels for each alternative, and then evaluating these alternatives as the final step. Consensus times would be reduced, and the trade-offs inherent in the problem would be made explicit.

The second problem listed may be the most intractable one for Gunthe $r$ and Vallery, particularly since present legislation requires substantial local participation for planning assistance. Local participation and involvement may be essential to the effective implementation of development programs, but the formulation of effective development programs may be beyond the capabilities of small local groups.

The is sue raised under point three, that a non-symmetric panel arrangement seems implied, has consequences that overlap into points one and two. Under a non-symmetric panel arrangement, experts in different fields are impanelled. If the se "experts" a re not highlyqualified individuals, then some improvement of the consensus reached might be attained if the issues to be 
decided were singled out to permit the use of symmetric panels. This, of course, would be consistent with the proposal previously advanced under point one, and might also offer some remedy for the problem mentioned under point two.

Finally, under point four, the questionnaire, while ostensibly being used to examine the potentials "... of public investments...to accelerate economic development, " clearly omits at least two important considerations. The first is the potential for competitive subsidization of industry, vigorously practiced in Alabama incidentally, and long advocated by Moes. ${ }^{2}$ Closely associated with this would be a consideration of alternative rates of return on such subsidies. Secondly, the questionnaire should probably focus some attention on the effects of proposed economic development on the local public fiscal capacity, for it seems clear that all economic development may not be beneficial in that respect. Further, this local public fiscal capacity is critical to the provision of local public services, a factor in future business location decisions.

In conclusion, it seems that the project outlined by Gunther and Vallery offers a usefulapplication of the Delphi technique, which is to be commended, but it appears to suffer from some technical difficulties. It is hoped that the se will be overcome before its implementation. In any event, the outcome of their experiment should be interesting. 


\section{FOOTNOTES}

lOlaf Helmer, Social Technology (Basic Books, Inc., 1966), p. 11.

2 John E. Moes, "The Subsidization of Industry by Local Communities in the South, " The Southern Economic Journal, XXVII, (October, 1961), pp. 187-193. 